\title{
EMS - NEW CHALLENGES AND NEW EXPECTATIONS
}

\author{
James Chebra \\ JFK Medical Center Emergency Medical Services, USA
}

Disaster Emerg Med J 2017; 2(2): 102-103

\section{Dear Editor,}

Here in the United States, EMS has been attacked in the media. Recently, several articles have appeared in national publications suggesting, even insisting that patients should call a taxi or use a service such as Uber instead of calling an ambulance. According to Leah Samuel published in Statnews, "For a trip to the ER, some are opting for Uber over an ambulance" citing the cost, reliability and unrestricted destination as the driving motivation (Fig. 1) [1]. This poses several interesting conundrums. The varying nature of emergency department visits spans both minor illnesses and injuries to major life-threatening events. The training that ambulance personnel receive is focused on the identification and immediate treatment. Taxi drivers do not possess such knowledge and it is a only matter of time before someone hails a taxi to a hospital for a stomach ache that turns out to be an inferior wall myocardial infarction. Mary Kekatos, writing for the Dailymail. com, recounts stories from Uber drivers requested to take a person suffering from anaphylactic shock to hospital [2]. In the United States approximately 17\%

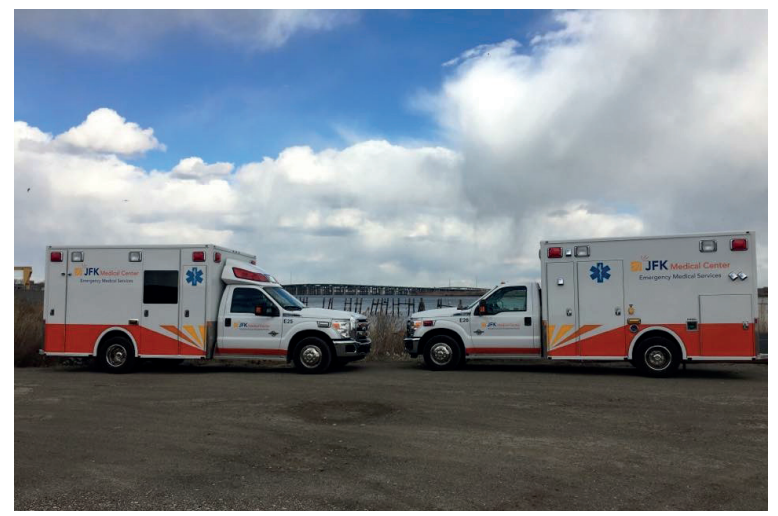

FIGURE 1. JFK Ambulances of total emergency department volume is generated from EMS transports and of these transports, approximately $39 \%$ of patients are admitted [3]. That means that, on average, $61 \%$ of patients transported by EMS to the emergency department do not require hospital admission. Although the appeal of lower costs and unrestricted destinations may appeal to the healthcare consumer, this comes at the price of safety. Many systems in the United States divide triage 911 calls into categories based on severity. In progressive systems, calls that are identified as non-life threatening are handled by medical taxis or other arrangements. Although calls to 911 is free, triage by a trained call-taker is free, and the responsible act of not putting EMS responders at risk for non-life threatening transports is of great value, this concept is new. It is progressive and outside of the comfort zone of many agencies.

The turmoil that the US health system has undergone and will continue to undergo will likely reinforce the idea of the healthcare consumer. As people become more financially responsible for their healthcare, cost has become the new chief complaint, while the quest for value in healthcare has come to EMS. Without the ability to show quality and meaningful improvement based on EMS intervention, the profession of EMS is destined for a tragic end.

At JFK EMS, we have taken the challenge of showing value in EMS to a new level. As part of our commitment to providing a quality service we have begun to track the Rapid Emergency Medical Score (REMS) (Fig. 2). This score is based on a patient's age, blood pressure, Pulse Oximetry, Mean Arterial Pressure and Glasgow Coma Score, with these values being correlated to a REMS score [4]. At JFK EMS, this score is calculated using the initial assess-

ADDRESS FOR CORRESPONDENCE:

James Chebra, JFK Medical Center Emergency Medical Services, USA, e-mail: jcheb@me.com 


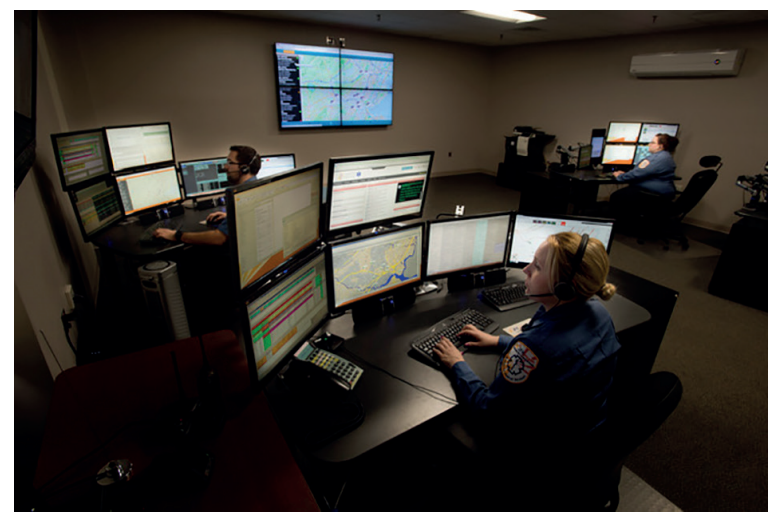

FIGURE 2. JFK control room

ment of vital signs while a comparison is taken from the last set of vital signs before turning over patient care to the hospital. The goal is to show quantifiable, empirical evidence that EMS makes a difference in patient outcomes. We are currently in the process of comparing the REMS score to the hospital length of stay. We currently have eighteen-months of data and from initial analysis, the top ten complaints that have shown a positive correlation to EMS treatment have been as follows: breathing problems; airway problems; drug overdose; altered level of consciousness; cardiac arrest; chest pain; trauma; haemorrhage; and pain. The study is ongoing.

Conflict of interest: None declared.

\section{REFERENCES}

1. Samuel L. For a trip to the ER, some are opting for Uber over an ambulance. https://www.statnews.com/2017/04/05/uber-lyft-emergency-room-ride/ (04.05.2017).

2. Kekatos M. Rise in passengers using Uber over ambulance to go to the hospital. http://www.dailymail.co.uk/ /article-4383990/index. html (04.05.2017).

3. Augustine JJ. Emergency Medical Services Arrivals, Admission Rates to the Emergency Department Analyzed. http://www.acepnow.com/ article/emergency-medical-services-arrivals-admission-rates-emergency-department-analyzed/3/ (04.05.2017).

4. Alter SM, Infinger A, Swanson D, et al. Evaluating clinical care in the prehospital setting: Is Rapid Emergency Medicine Score the missing metric of EMS? Am J Emerg Med. 2017; 35(2): 218-221, doi: 10.1016/j.ajem.2016.10.047, indexed in Pubmed: 27890300. 